\title{
Effect of Vitamin E and Alpha Lipoic Acid in Nonalcoholic Fatty Liver Disease: A Randomized, Placebo-Controlled, Open-Label, Prospective Clinical Trial (VAIN Trial)
}

\author{
Patrick P. Basu',2, Niraj J. Shah ${ }^{3}$, Mark M. Aloysius ${ }^{2 *}$, Robert S. Brown Jr.1 \\ ${ }^{1}$ Columbia University College of Physicians and Surgeons, New York, USA \\ ${ }^{2}$ King's County Hospital, New York, USA \\ ${ }^{3}$ James J. Peters VA Medical Center, Mount Sinai School of Medicine, New York, USA \\ Email: ${ }^{*}$ mark.aloysius5@gmail.com
}

Received 20 April 2014; revised 25 April 2014; accepted 2 May 2014

Copyright (C) 2014 by authors and Scientific Research Publishing Inc.

This work is licensed under the Creative Commons Attribution International License (CC BY). http://creativecommons.org/licenses/by/4.0/

c) (i) Open Access

\begin{abstract}
Objective: Antioxidants, including alpha lipoic acid (ALA) and vitamin E, are efficacious for the treatment of nonalcoholic fatty liver disease (NAFLD). The objective was to evaluate the effects of ALA and vitamin E alone or combined as therapy for patients with NAFLD and nonalcoholic steatohepatitis (NASH). Design: Placebo-controlled, open-label, prospective study in which patients with NAFLD and NASH were randomized to treatment with ALA $300 \mathrm{mg}(n=40)$, vitamin E 700 IU $(n=40)$, ALA $300 \mathrm{mg}$ plus vitamin E $700 \mathrm{IU}(n=40)$, or placebo $(n=35)$ daily for 6 months. Body mass index, homeostasis model assessment scores, fibrosis and steatosis markers, and diagnostic laboratory tests were assessed at baseline and at the end of the study. Results: Treatment with ALA and vitamin $E$ alone or in combination, improved inflammatory cytokine levels, steatosis scores, homeostasis model assessment scores, and triglyceride levels after 6 months relative to baseline. Conclusion: Alpha lipoic acid and vitamin E, either alone or in combination, were effective treatments for patients with NAFLD and NASH.
\end{abstract}

\section{Keywords}

Antioxidant, Liver Disease, Vitamin E, Alphalipoic Acid

${ }^{*}$ Corresponding author. 


\section{Introduction}

Nonalcoholic fatty liver disease (NAFLD) is the most common liver disease in the world and is rapidly becoming a global health problem [1]. In the United States, analysis of data from the Third National Health and Nutrition Examination Survey (NHANES III: 1988-1994) found the prevalence of NAFLD to be $16.4 \%$ in adults aged 20 to 74 [2]. Other reports have estimated the prevalence of NAFLD in children in the United States at 13\% to $14 \%$, while the prevalence of NAFLD in the morbidly obese has been estimated between $75 \%$ and $92 \%$ [1], which is not surprising considering its strong association with obesity and diabetes [2]. Indeed, NAFLD is characterized by a complex multifactorial etiology thought to involve pathologies including type 2 diabetes, obesity, hyperlipidemia, and inflammation [1]. However, NAFLD can also be caused by use of drugs (e.g., glucocorticoids, antiviral agents, and cocaine), nutritional etiologies (e.g., starvation, total parenteral nutrition), metabolic or genetic etiologies (e.g., lipodystrophy, Weber-Christian disease), inflammatory bowel disease, or environmental hepatotoxins [3]. Regardless of etiology, the disease spectrum of NAFLD ranges from "simple steatosis" to nonalcoholic steatohepatitis (NASH), NAFLD-associated cirrhosis, and end-stage liver disease [1].

While the pathogenesis of NAFLD is not completely understood, accumulation of lipids in hepatocytes, primarily in the form of triglycerides, is a requirement for the development of the disease [3], with fat accumulation in the liver exceeding approximately 5\% by weight [4]. Progression of NAFLD to more serious forms of liver disease (i.e., fibrosis, cirrhosis, liver cancer) [5] can be exacerbated by excess consumption of carbohydrates and lipids, which increase levels of fatty acids in the liver [6]. The liver is unable to esterify the surplus of free fatty acids into neutral triglycerides, ultimately leading to lipotoxicity and subsequent hepatocyte apoptosis [7]. Indeed, increased levels of free fatty acids have been associated with the degree of liver disease severity [8] and the major pathogenic event in the transition to NASH in patients with NAFLD is the increased release of free fatty acids from adipose tissue and increased peripheral lipolysis [9]. Insulin resistance plays a key role in this process, allowing for the surplus of fatty acids from adipose tissue and impaired peripheral glucose removal [10].

Increased rates of fatty acid oxidation in the liver may also lead to oxidative stress and the production of reactive oxygen species which may also cause hepatocellular damage and decreased cell viability [11]-[13]. Thus, targeting of pathways with antioxidants, such as alpha lipoic acid (ALA) and vitamin E, has been shown to improve liver pathology and laboratory endpoints in animal and human studies [14]-[23]. Alpha lipoic acid is a multifunctional antioxidant that prevents the release of free radicals [24] and lipid peroxidation [25]; restores insulin responsiveness [24]; regenerates other antioxidants, including vitamins E and C [24]; increases intracellular glutathione levels [24]; and inhibits cytokine-induced inflammation [26]. An animal model of hepatic steatosis showed decreased hepatic lipogenesis following treatment with ALA [16]. Vitamin E has been shown to reduce oxidative liver damage in an animal model [27] and to improve serum markers in children with NASH [18], serum indicators of oxidative stress in adults with NASH [19], and several markers of liver histology in the pioglitazone (Actos ${ }^{\circledR}$; Takeda Pharmaceutical Company Limited, Oksaka, Japan) versus vitamin E versus placebo for the treatment of nondiabetic patients with nonalcoholic steatohepatitis (PIVENS) clinical trial of NASH [17]. Because antioxidants such as ALA and vitamin E effectively improved liver histology and laboratory markers of liver disease in numerous studies, the efficacy of ALA $300 \mathrm{mg}$ and vitamin E $700 \mathrm{IU}$ in patients with NAFLD and NASH was evaluated in the randomized, placebo-controlled, open-label, prospective VAIN trial.

\section{Methods}

\subsection{Study Design}

This study was a randomized, placebo-controlled, open-label, prospective clinical trial conducted between February 2009 and November 2010 at North Shore Forest Hills Hospital. The study protocol was approved by the North Shore-Long Island Jewish Health System Institutional Review Board and was performed in accordance with the Declaration of Helsinki, the International Conference on Harmonization Good Clinical Practice Guidelines, and applicable local laws and regulations. Signed informed consent was obtained for each patient before study enrollment. Of 976 individuals interviewed, 175 refused and 637 did not meet the fulfillment criteria (Figure 1). All those recruited were compliant to follow up and there were no drop outs.

\subsection{Sample Size Calculation}

A total of 155 patients were estimated to enter this 4 arm parallel-design study. The probability was 80 percent 


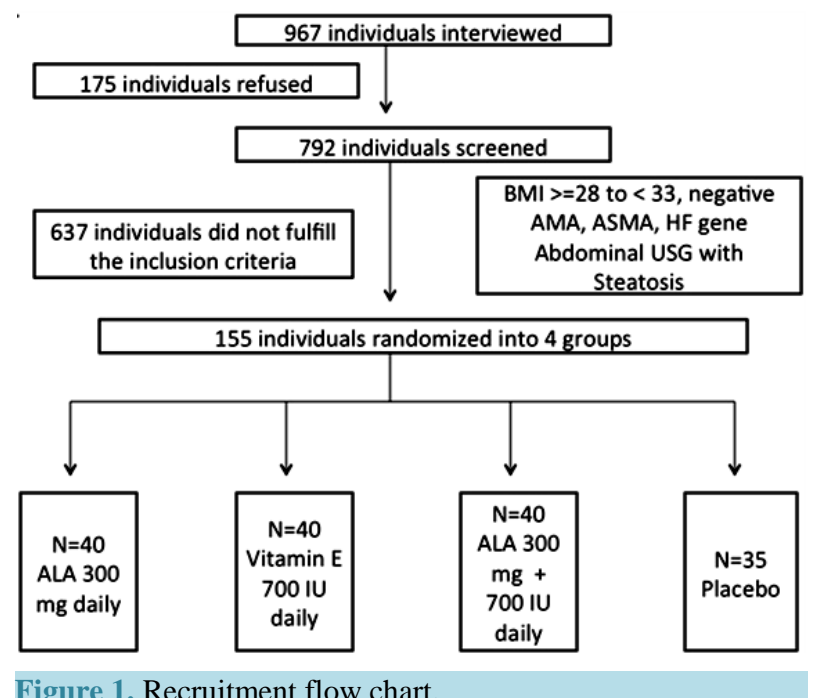

that the study would detect a treatment difference at a one-sided 0.05 significance level.

The sample size was calculated using well-recognized computer software called SampleSize (version 1.1.3, Epigenesis, Sheffield, UK) based on Julious SA et al. Statist (Med. 2004; 23:1921-1986). The envelopes were used for concealment but the randomization was based on random numbers generated by a computer. People uninvolved with the study were tasked with the randomization process. The codes were broken only during analysis. As the null hypothesis was that the new treatment would improve markers of non-alcoholic fatty liver disease, we were justified in using the one tailed $p$-value for significance. However, if we had assumed that the new treatment would be worse off or better, we would have used a p value with a 2-tailed significance. However, since this was a clinical trial, we could not justify violating a key principle in medicine is "do no harm" based on the fact that the interventional agents used in the study Vit E, alpha lipoic acid were FDA approved supplements with well established safety records, therefore a one tailed $p$ value for significance was justified.

\subsection{Patient Population}

Patients with NAFLD, NASH, and a body mass index of $\geq 28$ to $<33 \mathrm{~kg} / \mathrm{m}^{2}$ were included in the study. An abdominal ultrasound was used to diagnose the subjects. Patients had normal anti-mitochondrial antibody and antismooth muscle antibody levels and were negative for mutations at both alleles of the hemochromatosis gene. Exclusion criteria included diabetes, hepatitis B, hepatitis C, hypothyroidism, hyperthyroidism and syndromes with known insulin resistance. Patients consuming $>30 \mathrm{~g}$ of alcohol per day or receiving other medications, including herbs and supplements, were also excluded from the study.

\subsection{Treatment}

Patients were randomized (using sealed envelops) to receive placebo, ALA 300 mg, vitamin E 700 IU, or ALA $300 \mathrm{mg}$ plus vitamin E 700 IU for 6 months. Patients were advised to take ALA early morning, while Vitamin E was advised to take with the largest (with respect to fat intake) meal of the day. All groups received once a day oral medication. The medications were generic over the counter medications from the same pharmacy. Study participants were allowed to consume 1600 calories per day and to participate in moderate exercise (Walking 150 minutes per week at the rate of 100 steps per minute)

\subsection{Assessment of Clinical Parameters}

Body mass index was determined at baseline and following treatment for 6 months. Laboratory tests performed at baseline and at the end of the study determined fasting levels of plasma triglycerides, hemoglobin $\mathrm{A}_{1 \mathrm{c}}$, alanine aminotransferase (ALT), retinol-binding protein 4 (RBP-4), tumor necrosis factor $\alpha$ (TNF- $\alpha$ ), leptin, and adiponectin. The homeostasis model assessment (HOMA) score was calculated using the following formula: (fasting glucose $\times$ insulin)/22.5 [28]. Fibrosis and steatosis markers were determined by NASH FibroSURE ${ }^{\text {TM }}$ (Labora- 
tory Corporation of America, Burlington, NC) [29].

\subsection{Statistical Analysis}

Using SPSS version 21, statistical analysis with $\chi^{2}$ test was performed for categorical variables and a $p$ value of $\leq 0.05$ was considered to be statistically significant.

\section{Results}

\subsection{Patients and Baseline Characteristics}

A total of 155 patients were enrolled in the study. Forty patients were randomized to each treatment group of ALA $300 \mathrm{mg}$, vitamin E $700 \mathrm{IU}$, or ALA $300 \mathrm{mg}$ plus vitamin E $700 \mathrm{IU}$, and 35 patients were randomized to treatment with placebo. The demographics and baseline disease characteristics of the patients in the study are summarized in Table 1.

\subsection{Improvement in Inflammatory Cytokines and Steatosis Scores}

Treatment with the combination of ALA and vitamin E for 6 months resulted in a greater decrease in levels of the proinflammatory cytokine TNF- $\alpha$ compared with baseline versus placebo (82.6\% vs. $12.5 \%$, respectively; Figure 2). Treatment with either ALA or vitamin E alone also improved levels of TNF- $\alpha 59.3 \%$ and $61.9 \%$, respectively, compared with baseline. Treatment with the combination of ALA and vitamin E also improved steatosis scores from baseline to a greater extent than placebo (78.7\% vs. $8 \%$, respectively; Figure 3). Similar decreases were found with ALA or vitamin E monotherapy (75\% and 73.2\%, respectively).

\subsection{Improvement in Clinical Variables}

Homeostasis model assessment scores improved 76.1\% from baseline following treatment with the combination of ALA and vitamin E compared with 7.1\% for treatment with placebo, while either ALA or vitamin E monotherapy improved HOMA scores $54.3 \%$ and $53.7 \%$, respectively (Table 2). Triglyceride levels improved from baseline for all treatment groups: $56.4 \%, 34.4 \%$, and $37.5 \%$ for patients receiving the combination of ALA and vitamin E, ALA monotherapy, or vitamin E monotherapy, respectively, compared with $13.4 \%$ for the placebo group. Moreover, treatment with ALA plus vitamin E improved triglyceride levels to normal levels $(<160$ $\mathrm{mg} / \mathrm{dL})$.

Table 1. Patient demographics and baseline disease characteristics.

\begin{tabular}{cc}
\hline Characteristic & Patients $(\boldsymbol{n}=\mathbf{1 5 5})$ \\
\hline Age, mean \pm SD (range), y & $36 \pm 5(24-46)$ \\
Male:Female, $\mathrm{n}$ & $53: 102$ \\
Race, $n(\%)^{\mathrm{a}}$ & $63(40)$ \\
White & $45(29)$ \\
Black & $40(26)$ \\
Hispanic & $7(5)$ \\
Asian & $29(27.9-32)$ \\
BMI, mean (range), kg/m² & $4-9$ \\
Duration of liver disease, range, mo & $78(46-122)$ \\
ALT, mean (range), $\mathrm{U} / \mathrm{L}^{\mathrm{b}}$ & $113(98-133)$ \\
AST, mean (range), $\mathrm{U} / \mathrm{L}^{\mathrm{c}}$ & $123(116-143)$ \\
GGT, mean (range), $\mathrm{U} / \mathrm{L}^{\mathrm{d}}$ & $433(243-876)$ \\
Ferritin, mean (range), ng/mL & \\
\hline
\end{tabular}

ALT, alanine aminotransferase; AST, aspartate aminotransferase; BMI, body mass index; GGT, $\gamma$-glutamyltransferase; SD, standard deviation. ${ }^{\mathrm{a} D a t a}$ missing for 1 patient. ${ }^{b}$ Reference range, 10 - 40. ${ }^{\mathrm{c}}$ Reference range, 10 - 30. ${ }^{\mathrm{d}}$ Reference range, 2 - $30 .{ }^{\mathrm{e}}$ Reference range, 15 - 200. 


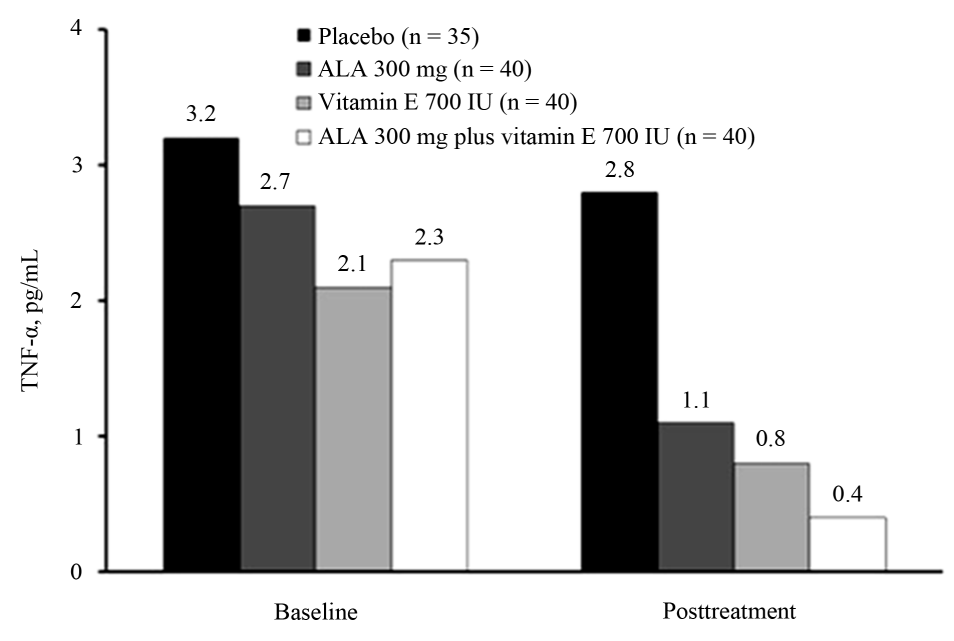

Figure 2. TNF- $\alpha$ levels at baseline and posttreatment. ALA, alpha lipoic acid; TNF- $\alpha$, tumor necrosis alpha.

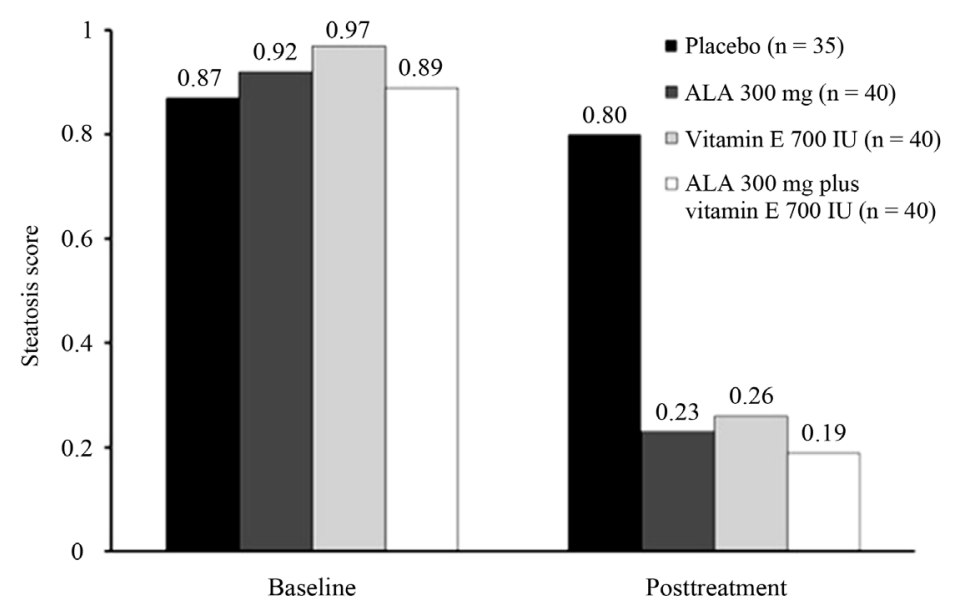

Figure 3. Steatosis scores at baseline and posttreatment. ALA, alpha lipoic acid.

Table 2. Patients' demographics and disease characteristics in different treatment (and placebo) arms.

\begin{tabular}{|c|c|c|c|c|}
\hline & Placebo $\quad n=35$ & VIT E $n=40$ & $n=40$ & VIT E + ALA \\
\hline Age, mean \pm SD (range) & $35 \pm 6(25-46)$ & $36 \pm 5(25-42)$ & $37 \pm 5(24-45)$ & $36 \pm 6(24-44)$ \\
\hline M:F & $10: 25$ & $14: 26$ & $13: 27$ & $16: 24$ \\
\hline Race: & & & & \\
\hline White & 18 & 14 & 18 & 13 \\
\hline Black & 10 & 12 & 10 & 13 \\
\hline Hispanic & 6 & 11 & 11 & 12 \\
\hline Asian & 1 & 3 & 1 & 2 \\
\hline BMI & $30(28.2$ - 31.6) & $29(28.9-32)$ & $28.5(27.9-31)$ & $30(28.7-31.8)$ \\
\hline Duration of Liver Disease & $\mathbf{n} / \mathbf{a}$ & $4-9$ & $5-9$ & $4-9$ \\
\hline ALT & 46 & 48 & 54 & 58 \\
\hline AST & 37 & 42 & 40 & 42 \\
\hline GGT & 17 & 24 & 25 & 24 \\
\hline FERRITIN & 27 & 203 & 187 & 203 \\
\hline
\end{tabular}


Treatment with the combination of ALA and vitamin E resulted in a $61.9 \%$ decrease from baseline in the levels of RBP-4, from $4.2 \mathrm{mg} / \mathrm{dL}$ before treatment to $1.6 \mathrm{mg} / \mathrm{dL}$ following treatment for 6 months (Table 2). Treatment with either ALA or vitamin E monotherapy resulted in a $46.2 \%$ or $46.5 \%$ decrease in RBP-4 levels from baseline, respectively. These reductions in RBP-4 were greater than that observed with placebo (11.4\% decrease from baseline). Leptin levels decreased $58.6 \%$ in patients receiving ALA and vitamin E, while patients receiving ALA or vitamin E monotherapy or placebo had a $44.8 \%, 46.9 \%$, or $35.3 \%$ decrease, respectively. Adiponectin levels increased $111.1 \%$ in patients receiving ALA and vitamin E combination therapy, $122.2 \%$ in patients receiving ALA monotherapy, and $91.7 \%$ in patients receiving vitamin E monotherapy, compared with only $50 \%$ in patients receiving placebo.

Treatment with the combination of ALA and vitamin E resulted in a 17.7\% decrease from baseline in hemoglobin $A_{1 c}$ levels, while treatment with ALA or vitamin $E$ monotherapy resulted in a $13.6 \%$ and $22 \%$ decrease in hemoglobin $A_{1 c}$ levels, respectively (as outlined in Table 3). Patients receiving placebo had a 3.3\% decrease in hemoglobin $A_{1 c}$ levels. Treatment with the combination of ALA and vitamin E resulted in a $44.8 \%$ decrease in ALT from baseline, while treatment with ALA monotherapy, vitamin E monotherapy, or placebo resulted in a 20.8\%, 40.7\%, or 30.4\% decrease in ALT from baseline, respectively. Fibrosis scores had little or no change regardless of treatment.

\section{Table 3. Clinical variables assessed before and after treatment.}

\begin{tabular}{|c|c|c|c|c|}
\hline Variable & $\begin{array}{l}\text { Placebo } \\
(n=35)\end{array}$ & $\begin{array}{l}\text { ALA } 300 \mathrm{mg} \\
\quad(n=40)\end{array}$ & $\begin{array}{l}\text { Vitamin E } 700 \mathrm{IU} \\
\quad(n=40)\end{array}$ & $\begin{array}{l}\text { ALA } 300 \mathrm{mg} \text { plus vitamin E } 700 \mathrm{IU} \\
\qquad(n=40)\end{array}$ \\
\hline \multicolumn{5}{|l|}{ HOMA score } \\
\hline Baseline & 4.2 & 4.6 & 4.1 & 4.6 \\
\hline Posttreatment & 3.9 & 2.1 & 1.9 & 1.1 \\
\hline Change from baseline & $-7.1 \%$ & $-54.3 \%$ & $-53.7 \%$ & $-76.1 \%$ \\
\hline \multicolumn{5}{|l|}{ Triglycerides, mg/dL } \\
\hline Baseline & 276 & 285 & 280 & 298 \\
\hline Posttreatment & 239 & 187 & 175 & 130 \\
\hline Change from baseline & $-13.4 \%$ & $-34.4 \%$ & $-37.5 \%$ & $-56.4 \%$ \\
\hline \multicolumn{5}{|l|}{ RBP-4, $\mu \mathrm{g} / \mathrm{mL}$} \\
\hline Baseline & 3.5 & 3.9 & 4.3 & 4.2 \\
\hline Posttreatment & 3.1 & 2.1 & 2.3 & 1.6 \\
\hline Change from baseline & $-11.4 \%$ & $-46.2 \%$ & $-46.5 \%$ & $-61.9 \%$ \\
\hline \multicolumn{5}{|l|}{ Leptin, ng/mL } \\
\hline Baseline & 34 & 29 & 32 & 29 \\
\hline Posttreatment & 22 & 16 & 17 & 12 \\
\hline Change from baseline & $-35.3 \%$ & $-44.8 \%$ & $-46.9 \%$ & $-58.6 \%$ \\
\hline \multicolumn{5}{|l|}{ Adiponectin, $\mu \mathrm{g} / \mathrm{mL}$} \\
\hline Baseline & 1.2 & 0.9 & 1.2 & 0.9 \\
\hline Posttreatment & 1.8 & 2.0 & 2.3 & 1.9 \\
\hline Change from baseline & $50 \%$ & $122.2 \%$ & $91.7 \%$ & $111.1 \%$ \\
\hline \multicolumn{5}{|l|}{ Hemoglobin $\mathrm{A}_{1 \mathrm{c}}, \%$} \\
\hline Baseline & 6.1 & 6.6 & 6.8 & 6.2 \\
\hline Posttreatment & 5.9 & 5.7 & 5.3 & 5.1 \\
\hline Change from baseline & $-3.3 \%$ & $-13.6 \%$ & $-22 \%$ & $-17.7 \%$ \\
\hline \multicolumn{5}{|l|}{ ALT, U/L } \\
\hline Baseline & 46 & 48 & 54 & 58 \\
\hline Posttreatment & 32 & 38 & 32 & 32 \\
\hline Change from baseline & $-30.4 \%$ & $-20.8 \%$ & $-40.7 \%$ & $-44.8 \%$ \\
\hline \multicolumn{5}{|l|}{ Fibrosis score } \\
\hline Baseline & 0.15 & 0.17 & 0.15 & 0.18 \\
\hline Posttreatment & 0.15 & 0.16 & 0.16 & 0.17 \\
\hline Change from baseline & $0 \%$ & $-5.9 \%$ & $6.7 \%$ & $-5.6 \%$ \\
\hline
\end{tabular}

ALA, alpha lipoic acid; ALT, alanine aminotransferase; RBP-4, retinol-binding protein 4; HOMA, homeostasis model assessment. 


\subsection{Adverse Events}

No adverse events were observed in any of the groups.

\section{Discussion}

The results of the VAIN trial suggest an additive effect of daily use of ALA and vitamin E for 6 months in patients with NAFLD and NASH compared with ALA and vitamin E monotherapy or treatment with placebo. To our knowledge, this is the first randomized, placebo-controlled, open-label, prospective clinical study comparing the efficacy of ALA and vitamin E alone or in combination in patients with NAFLD and NASH, and this study adds to the body of literature demonstrating the efficacy of vitamin E monotherapy [17] [21] or ALA and vitamin E combination therapy [20] for the treatment of patients with NAFLD and NASH.

The findings of the VAIN trial as they relate to markers of improved liver function and lipid levels are consistent with previous reports in the literature [17] [21]. There have been published reports comparing the efficacy of ALA or vitamin E monotherapy to other antioxidant and/or drug combinations or placebo. The reduction in ALT levels observed in this trial is comparable with the findings of the treatment of NAFLD in children (TONIC) trial [21]. In the TONIC trial, daily treatment with vitamin E 800 IU significantly reduced ALT levels from baseline after 24 or 48 weeks, although results were not sustained at 96 weeks. However, the findings of the TONIC trial are not directly comparable to this study due to the different study endpoints and the potential pathophysiologic differences in NAFLD and NASH in children and adults (i.e., pubertal changes, reduced or no ethanol exposure). Similar to the findings of this study, adults treated with vitamin E 800 IU in the phase 3, multicenter, randomized, placebo-controlled, double-blind PIVENS trial experienced significant improvement in serum ALT levels relative to baseline compared with placebo $(P=0.001)$ [17]. Treatment with vitamin $\mathrm{E}$ for 96 weeks compared with placebo also resulted in a significant rate of improvement in steatosis (54\% vs. $31 \%$; $P=0.005$ ), lobular inflammation ( $54 \%$ vs. $35 \%$; $P=0.02$ ), and hepatocellular ballooning ( $50 \%$ vs. $29 \%$; $P=0.01$ ) as determined by histological assessment. Because liver pathology was not an endpoint examined in this study, we cannot compare our findings with these published data. However, given a significant reduction in steatosis score and cytokine levels post-treatment indicates a reversal of the inflammatory process involved in the pathophysiology of non-alcoholic fatty liver disease.

Patients in the VAIN trial had a reduction in triglyceride levels following treatment with ALA or vitamin E monotherapy, an effect enhanced normal levels with a combination of ALA and vitamin E. While clinical trials of ALA in patients with NAFLD and NASH have not previously been conducted, there are limited data in experimental models demonstrating the potential for use of ALA in treatment of NAFLD and NASH. Our finding is comparable with that of a study of rats fed a high-fat diet, which demonstrated that treatment with ALA significantly reduced triglyceride levels compared with control animals or untreated animals on a high-fat diet [16].

The improvements in lipid profiles and HOMA scores in this study are similar to those of a study of patients with type 2 diabetes treated with ALA plus vitamin E, namely the lipid profiles of patients receiving ALA and vitamin $\mathrm{E}$ alone or in combination for 4 months were improved as were HOMA scores in patients receiving ALA monotherapy [20]. However, it is noteworthy that there were some differences between the demographics of the study groups (i.e., different countries, sex ratios, and ages), the duration and dosing of ALA and vitamin E [20], and that patients with diabetes were excluded from the VAIN trial.

One limitation of the VAIN trial was that liver biopsies were not performed at baseline or at the end of treatment. Histologic analysis is useful for determining the severity of liver disease, hepatocellular ballooning, and inflammation, especially because there are currently no validated biomarkers for determining response to treatment [17]. Furthermore, some of the clinical variables (e.g., aspartate aminotransferase, bilirubin [21]) examined by others were not included in this study. A second limitation is the shorter duration of this study compared with other studies. The TONIC study examined patients for 96 weeks and demonstrated that the significant changes in ALT levels observed at 24 and 48 weeks were reduced at 72 and 96 weeks because patients in the placebo group also experienced a reduction in ALT levels at 72 and 96 weeks [21]. This finding in the placebo group was attributed to increased patients' adherence to diet and exercise regimens and/or increased motivation because of study participation, factors not monitored in the VAIN trial. Additionally, this was a single-center study performed in New York, and the results may not fully extend to patient groups in other parts of the country or world, especially considering that a study analyzing data from NHANES III found that patients with NAFLD are more likely to be male and Mexican-American [2], both groups in the minority in this trial. It is also possible that res- 
ponses to treatment may differ by sex or ethnicity, an outcome not examined in this trial because of the small number of patients enrolled.

\section{Conclusion}

In conclusion, the VAIN trial demonstrates the efficacy of daily treatment with ALA and vitamin E for 6 months in patients with NAFLD and NASH. Large, randomized, multicenter clinical trials are needed to fully assess the efficacy and safety of ALA and vitamin E in patients with NAFLD and NASH. Due to the inconsistency in dosing and duration of treatment across published reports, a trial comparing ALA and vitamin E monotherapy or combination therapy for a longer length of time would be particularly beneficial. Based on the data presented in this report, ALA and vitamin E should be considered therapeutic options in patients with NAFLD and NASH.

\section{Conflict of Interest}

1) Guarantor of the article: P. Patrick Basu.

2) Specific author contributions: P. Patrick Basu and Robert S. Brown, Jr. were involved with planning and/or conducting the study. P. Patrick Basu, Mark M. Aloysius and Niraj J. Shah were involved with collecting and/or interpreting data. P. Patrick Basu, Mark M. Aloysius and Niraj J. Shah were involved with drafting the manuscript. P. Patrick Basu has approved the final draft submitted. Mark M. Aloysius has approved the final draft submitted. Niraj J. Shah has approved the final draft submitted. Robert S. Brown, Jr. has approved the final draft submitted.

3) Financial support: Editorial assistance was provided under the direction of the authors by Med Think SciCom with support from Salix Pharmaceuticals, Inc.

4) Potential competing interests: None.

\section{References}

[1] Erickson, S.K. (2009) Nonalcoholic Fatty Liver Disease. The Journal of Lipid Research, 50, S412-S416. http://dx.doi.org/10.1194/jlr.R800089-JLR200

[2] Lazo, M., Hernaez, R., Bonekamp, S., Kamel, I.R., Brancati, F.L., Guallar, E., et al. (2011) Non-Alcoholic Fatty Liver Disease and Mortality among US Adults: Prospective Cohort Study. BMJ, 343, d6891. http://dx.doi.org/10.1136/bmj.d6891

[3] Angulo, P. (2002) Nonalcoholic Fatty Liver Disease. The New England Journal of Medicine, 346, 1221-1231. http://dx.doi.org/10.1056/NEJMra011775

[4] Szczepaniak, L.S., Nurenberg, P., Leonard, D., Browning, J.D., Reingold, J.S., Grundy, S., et al. (2005) Magnetic Resonance Spectroscopy to Measure Hepatic Triglyceride Content: Prevalence of Hepatic Steatosis in the General Population. American Journal of Physiology_Endocrinology and Metabolism, 288, E462-E468. http://dx.doi.org/10.1152/ajpendo.00064.2004

[5] Bugianesi, E., Leone, N., Vanni, E., Marchesini, G., Brunello, F., Carucci, P., et al. (2002) Expanding the Natural History of Nonalcoholic Steatohepatitis: From Cryptogenic Cirrhosis to Hepatocellular Carcinoma. Gastroenterology, 123, 134-140. http://dx.doi.org/10.1053/gast.2002.34168

[6] Hijona, E., Hijona, L., Arenas, J.I. and Bujanda, L. (2010) Inflammatory Mediators of Hepatic Steatosis. Mediators of Inflammation, 2002, Article ID 837419. http://dx.doi.org/10.1155/2010/837419

[7] Ibrahim, S.H., Kohli, R. and Gores, G.J. (2011) Mechanisms of Lipotoxicity in NAFLD and Clinical Implications. Journal of Pediatric Gastroenterology and Nutrition, 53, 131-140.

[8] Nehra, V., Angulo, P., Buchman, A.L. and Lindor, K.D. (2001) Nutritional and Metabolic Considerations in the Etiology of Nonalcoholic Steatohepatitis. Digestive Diseases and Sciences, 46, 2347-2352. http://dx.doi.org/10.1023/A:1012338828418

[9] Schattenberg, J.M. and Schuppan, D. (2011) Nonalcoholic Steatohepatitis: The Therapeutic Challenge of a Global Epidemic. Current Opinion in Lipidology, 22, 479-488. http://dx.doi.org/10.1097/MOL.0b013e32834c7cfc

[10] Neuschwander-Tetri, B.A. (2010) Hepatic Lipotoxicity and the Pathogenesis of Nonalcoholic Steatohepatitis: The Central Role of Nontriglyceride Fatty Acid Metabolites. Hepatology, 52, 774-788. http://dx.doi.org/10.1002/hep.23719

[11] Gentile, C.L. and Pagliassotti, M.J. (2008) The Role of Fatty Acids in the Development and Progression of Nonalcoholic Fatty Liver Disease. The Journal of Nutritional Biochemistry, 19, 567-576. http://dx.doi.org/10.1016/j.jnutbio.2007.10.001 
[12] Solis-Herruzo, J.A., Garcia-Ruiz, I., Perez-Carreras, M. and Munoz-Yague, M.T. (2006) Non-Alcoholic Fatty Liver Disease: From Insulin Resistance to Mitochondrial Dysfunction. Revista Española de Enfermedades Digestivas, 98, 844-874. http://dx.doi.org/10.4321/S1130-01082006001100006

[13] Videla, L.A. (2009) Oxidative Stress Signaling Underlying Liver Disease and Hepatoprotective Mechanisms. World Journal of Hepatology, 1, 72-78. http://dx.doi.org/10.4254/wjh.v1.i1.72

[14] Abdelmalek, M.F., Angulo, P., Jorgensen, R.A., Sylvestre, P.B. and Lindor KD. (2001) Betaine, a Promising New Agent for Patients with Nonalcoholic Steatohepatitis: Results of a Pilot Study. The American Journal of Gastroenterology, 96, 2711-2717. http://dx.doi.org/10.1111/j.1572-0241.2001.04129.x

[15] Baumgardner, J.N., Shankar, K., Hennings, L., Albano, E., Badger, T.M. and Ronis, M.J. (2008) N-Acetylcysteine Attenuates Progression of Liver Pathology in a Rat Model of Nonalcoholic Steatohepatitis. Journal of Nutrition, 138, 1872-1879.

[16] Park, K.G., Min, A.K., Koh, E.H., Kim, H.S., Kim, M.O., Park, H.S., et al. (2008) Alpha-Lipoic Acid Decreases Hepatic Lipogenesis through Adenosine Monophosphate-Activated Protein Kinase (AMPK)-Dependent and AMPK-Independent Pathways. Hepatology, 48, 1477-1486. http://dx.doi.org/10.1002/hep.22496

[17] Sanyal, A.J., Chalasani, N., Kowdley, K.V., McCullough, A., Diehl, A.M., Bass, N.M., et al. (2010) Pioglitazone, Vitamin E, or Placebo for Nonalcoholic Steatohepatitis. The New England Journal of Medicine, 362, 1675-1685. http://dx.doi.org/10.1056/NEJMoa0907929

[18] Lavine, J.E. (2000) Vitamin E Treatment of Nonalcoholic Steatohepatitis in Children: A Pilot Study. The Journal of Pediatrics, 136, 734-738. http://dx.doi.org/10.1016/S0022-3476(00)05040-X

[19] Kawanaka, M., Mahmood, S., Niiyama, G., Izumi, A., Kamei, A., Ikeda, H., et al. (2004) Control of Oxidative Stress and Reduction in Biochemical Markers by Vitamin E Treatment in Patients with Nonalcoholic Steatohepatitis: A Pilot Study. Hepatology Research, 29, 39-41. http://dx.doi.org/10.1016/j.hepres.2004.02.002

[20] de Oliveira, A.M., Rondo, P.H., Luzia, L.A., D’Abronzo, F.H. and Illison, V.K. (2011) The Effects of Lipoic Acid and Alpha-Tocopherol Supplementation on the Lipid Profile and Insulin Sensitivity of Patients with Type 2 Diabetes Mellitus: A Randomized, Double-Blind, Placebo-Controlled Trial. Diabetes Research and Clinical Practice, 92, 253-260. http://dx.doi.org/10.1016/j.diabres.2011.02.010

[21] Lavine, J.E., Schwimmer, J.B., Van Natta, M.L., Molleston, J.P., Murray, K.F., Rosenthal, P., et al. (2011) Effect of Vitamin E or Metformin for Treatment of Nonalcoholic Fatty Liver Disease in Children and Adolescents: The TONIC Randomized Controlled Trial. Journal of the American Medical Association, 305, 1659-1668. http://dx.doi.org/10.1001/jama.2011.520

[22] Min, A.K., Kim, M.K., Seo, H.Y., Kim, H.S., Jang, B.K., Hwang, J.S., et al. (2010) Alpha-Lipoic Acid Inhibits Hepatic PAI-1 Expression and Fibrosis by Inhibiting the TGF-Beta Signaling Pathway. Biochemical and Biophysical Research Communications, 393, 536-541. http://dx.doi.org/10.1016/j.bbrc.2010.02.050

[23] Morsy, M.A., Abdalla, A.M., Mahmoud, A.M., Abdelwahab, S.A. and Mahmoud, M.E. (2011) Protective Effects of Curcumin, Alpha-Lipoic Acid, and N-Acetylcysteine against Carbon Tetrachloride-Induced Liver Fibrosis in Rats. Journal of Physiology and Biochemistry, 68, 29-35.

[24] Packer, L. and Cadenas, E. (2011) Lipoic Acid: Energy Metabolism and Redox Regulation of Transcription and Cell Signaling. Journal of Clinical Biochemistry and Nutrition, 48, 26-32. http://dx.doi.org/10.3164/jcbn.11-005FR

[25] Androne, L., Gavan, N.A., Veresiu, I.A. and Orasan, R. (2000) In Vivo Effect of Lipoic Acid on Lipid Peroxidation in Patients with Diabetic Neuropathy. In Vivo, 14, 327-330.

[26] Shay, K.P., Moreau, R.F., Smith, E.J., Smith, A.R. and Hagen, T.M. (2009) Alpha-Lipoic Acid as a Dietary Supplement: Molecular Mechanisms and Therapeutic Potential. Biochimica et Biophysica Acta (BBA)—General Subjects, 1790, 1149-1160. http://dx.doi.org/10.1016/j.bbagen.2009.07.026

[27] Parola, M., Leonarduzzi, G., Biasi, F., Albano, E., Biocca, M.E., Poli, G., et al. (1992) Vitamin E Dietary Supplementation Protects against Carbon Tetrachloride-Induced Chronic Liver Damage and Cirrhosis. Hepatology, 16, $1014-1021$. http://dx.doi.org/10.1002/hep.1840160426

[28] Angulo, P., Hui, J.M., Marchesini, G., Bugianesi, E., George, J., Farrell, G.C., et al. (2007) The NAFLD Fibrosis Score: A Noninvasive System That Identifies Liver Fibrosis in Patients with NAFLD. Hepatology, 45, 846-854. http://dx.doi.org/10.1002/hep.21496

[29] Neff, G.W., O’Brien, C.B., Reddy, K.R., Bergasa, N.V., Regev, A., Molina, E., et al. (2002) Preliminary Observation with Dronabinol in Patients with Intractable Pruritus Secondary to Cholestatic Liver Disease. The American Journal of Gastroenterology, 97, 2117-2119. http://dx.doi.org/10.1111/j.1572-0241.2002.05852.x 